\title{
Prenatal nicotine exposure induces depression-like behavior in adolescent female rats via modulating neurosteroid in the hippocampus
}

\author{
CHONG ZHANG ${ }^{1 *}$, SI-JING FAN ${ }^{2 *}$, AN-BANG SUN ${ }^{3,4}$, ZHEN-ZHEN LIU $^{2,3}$ and LIAN LIU ${ }^{2,3}$ \\ ${ }^{1}$ Department of Pharmacy, Xiangyang No. 1 People's Hospital, Hubei University of Medicine, Xiangyang, Hubei 441000; \\ ${ }^{2}$ Department of Pharmacology, Medical School of Yangtze University; ${ }^{3}$ Laboratory of Neuronal and Brain Disease Modulation, \\ Yangtze University; ${ }^{4}$ Department of Anatomy, Medical School of Yangtze University, Jingzhou, Hubei 434023, P.R. China
}

Received July 30, 2018; Accepted February 28, 2019

DOI: $10.3892 / \mathrm{mmr} .2019 .10105$

\begin{abstract}
Prenatal nicotine exposure (PNE) is closely related to depression in offspring. However, the underlying mechanism is still unclear. We hypothesized that neurosteroid in the hippocampus may mediate PNE-induced depression-like behaviors. Nicotine was subcutaneously administered $(1.0 \mathrm{mg} / \mathrm{kg})$ to pregnant rats twice daily from gestational day (GD) 9 to 20. In adolescent offspring, PNE significantly increased immobility time and decreased the sucrose preference in female rats. The numbers of hippocampal neurons declined in the CA3 and DG regions. Steroidogenic acute regulatory protein (StAR) expression was suppressed in female rats. In fetal offspring, the neuronal numbers of CA3 regions in PNE female fetal hippocampal were significantly decreased, accompanied by the enhanced content of corticosterone and StAR expression. These data indicated that PNE induced depression-like behavior in adolescent female rats via the regulation of neurosteroid levels in the hippocampus.
\end{abstract}

\section{Introduction}

Depression is a group of moods or affective disorders characterized by significant and persistent depression or loss of interest.

Correspondence to: Dr Lian Liu, Department of Pharmacology, Medical School of Yangtze University, 1 Nan Huan Road, Jingzhou, Hubei 434023, P.R. China

E-mail: liulian@yangtzeu.edu.cn

${ }^{*}$ Contributed equally

Abbreviations: PNE, prenatal nicotine exposure; GD, gestational day; StAR, steroidogenic acute regulatory protein; P450scc, P450 side-chain cleavage enzyme; GAPDH, glyceraldehyde-3-phosphate dehydrogenase; CORT, corticosterone; OFT, open field test; SPT, sucrose preference test

Key words: nicotine, steroidogenic acute regulatory protein, hippocampus, neurosteroid
According to the World Health Organization, approximately 145 million individuals worldwide suffer from depression (1). The prevalence of depression has reached 2.4-5.5\% in China (2). The pathogenesis of depression is complex, and the underlying mechanisms have not been completely clarified. The occurrence of depression is associated with individual susceptibility even in the same external environment. An increasing number of scholars have paid attention to the influence of poor uterine environment (smoking, drugs and maternal diseases) on the susceptibility to depression in offspring (3-5).

According to the Centers for Disease Control and Prevention (Atlanta, GA, USA), $12.3 \%$ of pregnant women have tobacco smoking experiences (6). Several clinical studies have observed an increase in mood disorders in children exposed to tobacco in utero (7). A study on a birth cohort of 1,265 children born in New Zealand found that those children whose mothers smoked during pregnancy had a higher rate of psychiatric symptoms (depression) (8). Children of mothers who smoked during pregnancy demonstrated symptoms of depression (9), indicating that maternal smoking is capable of altering the trajectory of neurobehavioral development in offspring. All of this evidence suggests that depression may have the origin of intrauterine development, and the susceptibility to depression is closely related to smoking exposure during pregnancy of the mothers.

Neurosteroids are a type of neurological molecules in the central nervous system, which are involved in physiological cortical functions and various pathological phenomena. Previous research indicates the endogenous overproduction of neurosteroids in several neuropsychiatric disorders, including depression $(10,11)$. Neurosteroids are generated from cholesterol by a series of steps which include the trafficking of cholesterol to the outer mitochondrial membrane by a process requiring the involvement of steroidogenic acute regulatory protein (StAR), and the conversion to pregnenolone by $\mathrm{P} 450$ side-chain cleavage enzyme (P450scc) $(12,13)$. The level of neurosteroids can be modulated by pharmacological agents such as corticosterone (14) and alcohol (15).

Nicotine is the major psychoactive substance among more than 4,000 different chemicals found in tobacco (16). Research has demonstrated that nicotine from inhaled smoke can easily 
distribute to the fetus through the placental barrier (17). Prenatal nicotine exposure (PNE) has been found to decrease cell numbers and/or cell size in the hippocampus (18) and alter learning and memory in rat offspring $(19,20)$. In our previous study, we demonstrated that PNE suppresses fetal adrenal steroidogenesis via restraining the expression of steroidogenic enzymes (21). Thus, we further hypothesized that depression-like behaviors in adult rats induced by maternal nicotine exposure may be related to the alteration of neurosteroid levels in the hippocampus.

The present study was designed to verify that PNE-induced behavior alterations in offspring are associated with changes in hippocampal morphology and the expression of StAR/P450scc before and after birth. Our findings may provide more evidence of the effects of PNE on the development of depression-like behaviors in adolescent offspring, and further elucidate the potential mechanisms responsible for nicotine developmental neurotoxicity via modulating neurosteroid levels in the hippocampus.

\section{Materials and methods}

Chemicals and reagents. Nicotine was purchased from Sigma-Aldrich (Merck KGaA, Darmstadt, Germany). A rat corticosterone (CORT) assay kit was obtained from Assaypro (St. Charles, MO, USA). The RNA-Solv reagent kit was provided by Omega Bio-Tek Inc. (Norcross, GA, USA). The reverse transcription (RT) reagent kit and RT-quantitative polymerase chain reaction (RT-qPCR) kit were purchased from Takara Biotechnology Co., Ltd. (Dalian, China). The protein detection kit was from Bio-Rad Laboratories, Inc. (Hercules, CA, USA). Polyclonal antibodies against rat StAR were obtained from Santa Cruz Biotechnology, Inc. (Dallas, TX, USA), and antibodies against P450scc were obtained from Boster Biological Technology (Pleasanton, CA, USA). The oligonucleotide primers of rat StAR and P450scc were synthesized by Sangon Biotech Co., Ltd. (Shanghai, China). Isoflurane was purchased from Baxter Healthcare Co. (Deerfield, IL, USA).

Animals and treatment. A total of 25 pathogen-free Wistar rats weighing 180-220 g (20 females) and 260-300 g (5 males) were obtained from the Experimental Center of Hubei Medical Scientific Academy (Licence no. 2008-0004; animal no. 42000600002575; 2014-02-13, Hubei, China). The animals were allowed to acclimate for 1 week prior to experimentation under controlled temperature $\left(24 \pm 2^{\circ} \mathrm{C}\right)$, humidity $(50-55 \%)$ and light conditions (12:12-h light/dark cycle; 07:00-19:00 light phase) and were provided access to rodent chow and water ad libitum. All animal studies were performed at the Animal Center of Yangtze University, accredited by the Association for Assessment and Accreditation of Laboratory Animal Care International (AAALAC International; Frederick, MD, USA). All animal experiments were approved by the Ethics Committee of Yangtze University, School of Medicine (Jingzhou, China) and performed in accordance with the Guidelines for the Care and Use of Laboratory Animals of Yangtze University.

The animals were treated as previously described (22). The neural tube of the rats takes shape between embryonic days (ED) 10.5 and ED11 $(23,24)$. The hippocampal primordium of the rats is composed of neuroepithelium that appears in the dorsomedial telencephalon by ED14 (25). Two female rats were placed together with one male rat overnight for mating. Gestational day (GD) 0 of mating was determined by the appearance of sperm in a vaginal smear. Nicotine was subcutaneously administered $(1.0 \mathrm{mg} / \mathrm{kg})$ to pregnant rats twice per day from GD9 to GD20, and the control group was sham-treated with the same volume of saline solution. We administered nicotine from GD9 because we focused on the development of the fetal rat hippocampus during the period of rat neural tube development. Thus, the fetal rats were exposed to nicotine at the day before the neural tube development. On GD20, a portion of the pregnant rats were anesthetized with isoflurane and then fetuses were collected by Caesarean section. Each feto-placental unit was removed quickly from the uterus. Each group contained 6 pregnant rats, and the litter size of each pregnant rat was from 8 to 14 . The fetal brains were quickly removed and placed in a chilled brain block for the dissection of the hippocampus, as previously reported (26). Another 4 pregnant rats in each group were allowed to deliver spontaneously at term, and a total of 8-12 pups from each litter were kept (male:female $=1: 1$ ). On postnatal day (PD) 21, the pups were separated from their mothers. The offspring from 4 maternal rats were combined in one group. Sucrose preference test and open field test were performed from PD29 to PD35. After the last test on PD35, the animals were anesthetized with isoflurane. Eight adolescent rats (male:female=1:1) in each group were randomly selected to be perfused with saline and then with ice-cold $4 \%$ phosphate-buffered paraformaldehyde. Brains were fixed in $4 \%$ paraformaldehyde overnight at a controlled temperature $\left(24 \pm 2^{\circ} \mathrm{C}\right)$. The remaining brain were dissected and stored at $-80^{\circ} \mathrm{C}$ for subsequent use.

Sucrose preference test. According to the previous study (27), rats were acclimated to two bottles of sucrose solution $(1 \%$, $\mathrm{w} / \mathrm{v}$ ) for $24 \mathrm{~h}$, and one bottle of sucrose solution was replaced with water for $24 \mathrm{~h}$. On the third day, rats were deprived of water and food for $23 \mathrm{~h}$, and during the last $1 \mathrm{~h}$, rats were given free access to two pre-weighed bottles of solution. The mass of sucrose and water consumed in both bottles was recorded in grams. The sucrose preference rate was calculated using the following formula: Sucrose preference rate $=$ sucrose consumption $(\mathrm{g}) /[$ water consumption $(\mathrm{g})+$ sucrose consumption $(\mathrm{g})]$.

Open field test. According to the previous study (28), the open field test was used to evaluate the locomotor activity and exploratory behavior. A plywood black wall box of $120 \times 90 \times 30 \mathrm{~cm}$ was divided into 25 equal squares. Each rat was placed individually in the center of the apparatus at the beginning of the test, and observed for $5 \mathrm{~min}$. The behaviors of rats were recorded. When both hind legs crossed the line of a square, the rat was considered to have crossed on to the next square (crossing). When the forelegs were lifted from the floor, the animal scored one point. At the end of each open field test, the cage was carefully cleaned with $75 \%$ ethanol and purified water prior to the test for the next rat, ensuring no possible inference on spontaneous behavior for the next animal.

Histopathology. Fixed rat brain samples were progressively dehydrated with ethanol and dimethylbenzene, embedded 
Table I. Oligonucleotide primers and PCR conditions.

\begin{tabular}{lllllr}
\hline & & & & \multicolumn{2}{c}{ Annealing } \\
\cline { 3 - 6 } Genes & \multicolumn{1}{c}{ Forward primer (5'-3') } & \multicolumn{1}{c}{ Reverse primer $\left(5^{\prime}-3^{\prime}\right)$} & $\begin{array}{c}\text { Product } \\
(\mathrm{bp})\end{array}$ & $\begin{array}{c}\text { Temperature } \\
\left({ }^{\circ} \mathrm{C}\right)\end{array}$ & $\begin{array}{r}\text { Time } \\
(\mathrm{sec})\end{array}$ \\
\hline StAR & GGGAGATGCCTGAGCAAAGC & 5GCTGGCGAACTCTATCTGGGT & 188 & 65 & 30 \\
P450scc & GCTGCCTGGGATGTGATTTC & GATGTTGGCCTGGATGTTCTTG & 156 & 68 & 30 \\
GAPDH & GCAAGTTCAACGGCACAG & GCCAGTAGACTCCACGACA & 107 & 63 & 30
\end{tabular}

PCR, polymerase chain reaction; StAR, steroidogenic acute regulatory protein; P450scc, P450 side-chain cleavage enzyme; GAPDH, glyceraldehyde-3-phosphate dehydrogenase.

with paraffin, and subjected to sectioning (5 $\mu \mathrm{m}$ thickness). A total of four sections were assessed in each brain for histological assay. Neuronal cell loss was assessed via Nissl staining in $1 \%$ thionin at $37^{\circ} \mathrm{C}$ for $10 \mathrm{~min}$. Neuronal cell counting in the hippocampus areas CA1, CA3 and DG was conducted using a light microscope (magnification, $\mathrm{x} 40$; DMi8; Leica Microsystems GmBH, Wetzlar, Germany). For quantitative assessment of brain injury, cells were counted in 5 non-overlapping regions of interest (ROI) in the hippocampus, as previously described (29).

Immunohistochemistry. The expression of StAR and P450scc were assessed by immunohistochemistry. Brains fixed in $4 \%$ paraformaldehyde overnight as previously described, and then transferred to $30 \%$ sucrose in $0.1 \mathrm{~mol} / \mathrm{l}$ phosphate buffer ( $\mathrm{pH}$ 7.4). Sections $(5 \mu \mathrm{m})$ were treated with $3 \% \mathrm{H}_{2} \mathrm{O}_{2}$ for $10 \mathrm{~min}$ and blocked with $10 \%$ normal goat serum (Vector Laboratories, Inc., Burlingame, CA, USA) for $2 \mathrm{~h}$ at room temperature. The sections were incubated with anti-StAR (1:50; sc-25806, Santa Cruz Biotechnology, Inc.) and anti-P450scc primary antibodies (1:50; PB0983, Boster Biological Technology) overnight at $4^{\circ} \mathrm{C}$. The sections were then washed in PBS-0.1\% Triton X (PBS-TX) and incubated with biotinylated goat anti-rabbit secondary antibodies (1:200; PK-6101, Vector Laboratories, Inc.) for $1 \mathrm{~h}$ at room temperature. Following two washes in PBS-TX, the sections were incubated with horseradish peroxidase (HRP)-avidin-biotin complex reagent (VECTASTAIN ${ }^{\circledR}$ ABC kit; PK-6101, Vector Laboratories, Inc.) in PBS-TX for $1 \mathrm{~h}$ at room temperature, and then washed in PBS-TX and reacted in 3,3'-diaminobenzidine peroxidase substrate (Vector Laboratories, Inc.) for $10 \mathrm{~min}$ at room temperature. Following immunostaining, the sections were mounted, counterstained with $10 \%$ hematoxylin for $5 \mathrm{~min}$ at room temperature, and washed in $0.5 \%$ ammonium hydroxide for $10 \mathrm{~min}$. Sections were left to dry on the bench at room temperature, and then covered with a coverslip. A minimum of five random fields per section were examined at a magnification of x40 (DMi8) and analyzed using a Medical Color Image Analysis System (HPIAS-2000 version 2.0; Wuhan Qianli Technical Imaging Co., Ltd., Wuhan, China).

Corticosterone (CORT) detection. Fetal hippocampus was homogenized in $20 \%$ ethanol in PBS, and then centrifuged at $1,200 \times \mathrm{g}$ at $4^{\circ} \mathrm{C}$ for $5 \mathrm{~min}$. The supernatants were collected for CORT analysis, and the pellets were resuspended in $1 \mathrm{~N}$
$\mathrm{NaOH}$ to measure protein content (21). The cross-reactivity for the CORT ELISA, as defined by the manufacturer, was $2 \%$ for progesterone and $2 \%$ for aldosterone. CORT content was expressed relative to hippocampus protein measured using the total protein detection kit.

$R N A$ extraction and $R T-q P C R$. Total RNA was isolated from fetal hippocampus using TRIzol ${ }^{\circledR}$ reagent (Thermo Fisher Scientific, Inc., Waltham, MA, USA) according to the manufacturer's protocol. The first strand of cDNA was produced using a Primescript ${ }^{\mathrm{TM}} \mathrm{RT}$ reagent kit with genomic DNA Eraser (Takara Biotechnology Co., Ltd.) according to the manufacturer's protocol. mRNA was quantified by qPCR using SYBR Premix Ex Taq II (Takara Biotechnology Co., Ltd.) and a ABI 7500 Real-Time PCR detection system (Applied Biosystems; Thermo Fisher Scientific, Inc.) was performed according to a previously described method (30). Using $20 \mu 1$ of the reaction system, the reaction conditions were: Pre-denaturing at $95^{\circ} \mathrm{C}$ for $30 \mathrm{sec}$, and 45 cycles of $95^{\circ} \mathrm{C}$ for $5 \mathrm{sec}$ and $63-68^{\circ} \mathrm{C}$ for $30 \mathrm{sec}$ (Table I). Primers were designed using Primer Premier 5.0 (Premier Biosoft International, Palo Alto, CA, USA), and their sequences are presented in Table I. The mRNA level of the housekeeping gene glyceraldehyde-3-phosphate dehydrogenase (GAPDH) was measured and used as a quantitative control. The $2^{-\Delta \Delta \mathrm{Cq}}$ method was used to quantify the expression of target genes (31). Experiments were repeated twice.

Western blot analysis. Proteins were prepared using a protein extraction kit (radioimmunoprecipitation assay lysis buffer; Beyotime Institute of Biotechnology, Wuhan, China). Total tissue extracts were quantified using a Bicinchoninic Acid Protein Assay kit (Beyotime Institute of Technology) with Synergy 4 (BioTek Instruments, Inc., Winooski, VT, USA). The samples $(50 \mu \mathrm{g} /$ lane) were separated via $12 \%$ SDS-PAGE. The proteins were transferred onto a nitrocellulose membrane. The membranes were blocked with $5 \%$ non-fat dry milk solution for $2 \mathrm{~h}$ at room temperature and incubated overnight at $4{ }^{\circ} \mathrm{C}$ with primary antibodies against StAR (1:500), P450scc (1:500) and $\beta$-actin (1:2,000; cat. no. 4970, Cell Signaling Technology, Inc., Danvers, MA, USA). Finally, the membrane was incubated for $45 \mathrm{~min}$ at room temperature with an HRP-conjugated goat anti-rabbit immunoglobulin G secondary antibody $(1: 2,000$; sc-2004, Santa Cruz Biotechnology, Inc.). Specific bands were detected using a BeyoECL Star enhanced chemiluminescence 

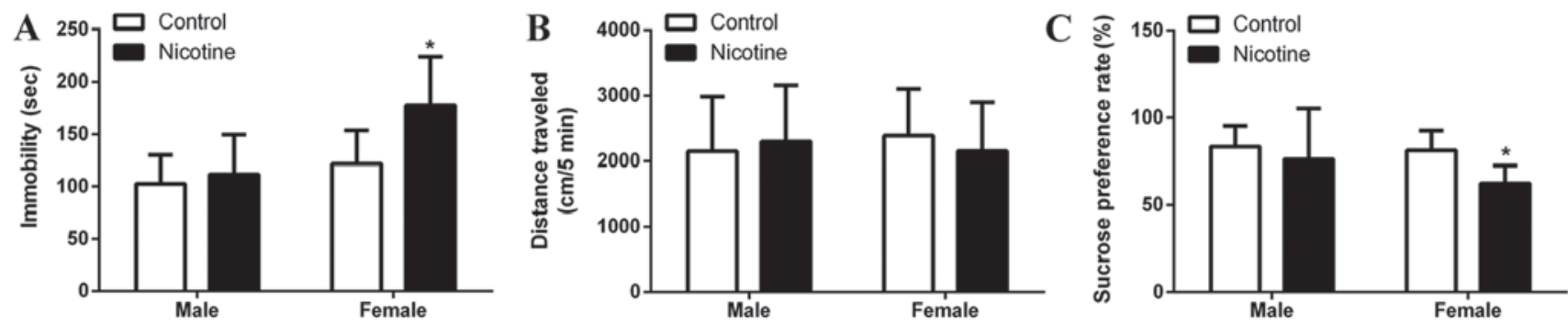

Figure 1. PNE induces depressive-like symptoms in adolescent rats. Pregnant rats were subcutaneously administered $1.0 \mathrm{mg} / \mathrm{kg}$ nicotine twice per day from GD9 to 20. The pregnant rats were allowed to deliver spontaneously at term. The offspring were subjected to OFT and SPT at PD29. (A) Immobility time in OFT. (B) Horizontal movement in OFT. (C) Sucrose preference in SPT. The data are expressed as mean \pm standard error of the mean; $n=16-20$ offsprings; ${ }^{*} \mathrm{P}<0.05$ vs. control. PNE, prenatal nicotine exposure; GD, gestational day; OFT, open field test; SPT, sucrose preference test; PD, postnatal day.
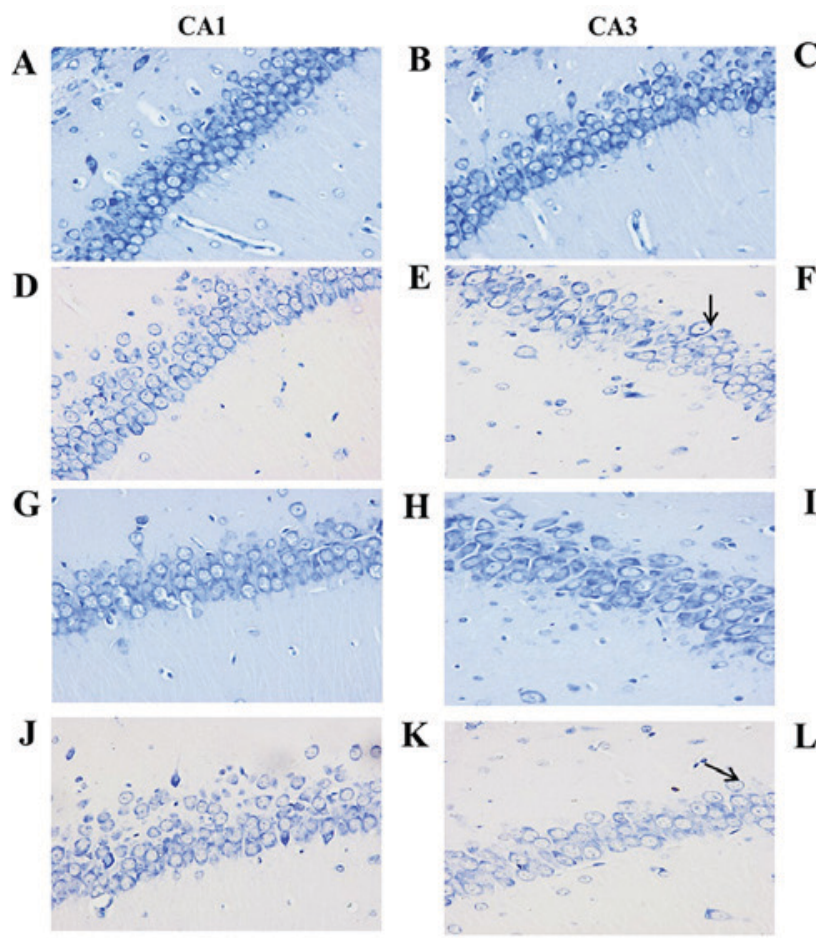

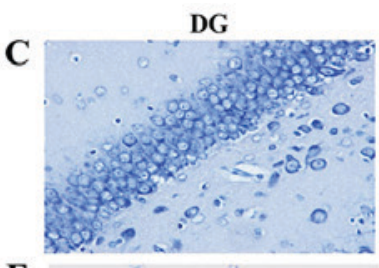

F
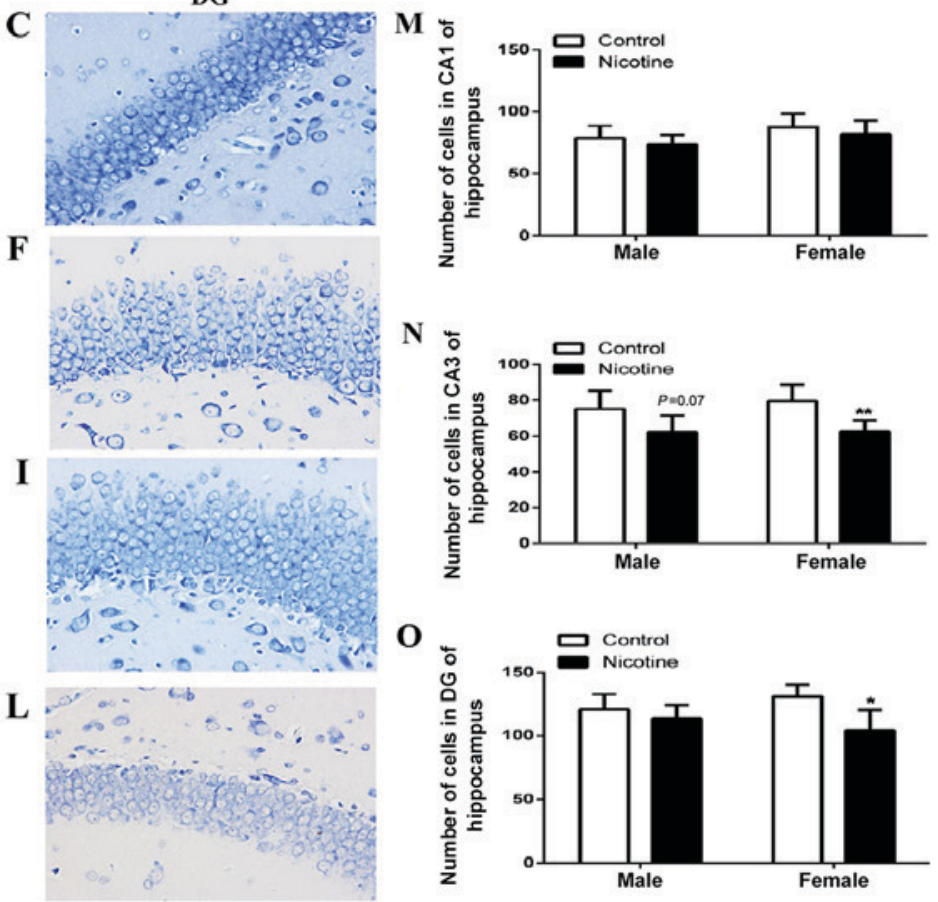

Figure 2. Morphological evaluation of Nissl staining in CA1, CA3 and DG regions of the hippocampus in adolescent rats. (A-C) Morphology of the hippocampus in male control group (magnification, $\mathrm{x} 40$ ). (D-F) Morphology of the hippocampus in male PNE (magnification, $\mathrm{x} 40$ ). (G-I) Morphology of the hippocampus in female control group (magnification, x40). (J-L) Morphology of the hippocampus in female PNE (magnification, x40). Arrows indicate the dissolved Nissl bodies. (M-O) The number of cells in CA1, CA3 and DG of rat hippocampus. Five sections of each group were selected and five random fields of each section were scored. The data are expressed as mean \pm standard error of the mean; $\mathrm{n}=4$ offsprings; ${ }^{*} \mathrm{P}<0.05,{ }^{* * *} \mathrm{P}<0.01 \mathrm{vs}$. control. $\mathrm{PNE}$, prenatal nicotine exposure.

kit (Beyotime Institute of Biotechnology). Band densities were analyzed using Image-Pro Plus 6.0 (Media Cybernetics, Inc., Rockville, MD, USA).

Statistical analysis. Data were analyzed using SPSS 17 (SPSS Inc., Chicago, IL, USA) and GraphPad Prism 6 (GraphPad Software, La Jolla, CA, USA). Data are presented as the mean \pm standard error of the mean of at least three experiments. Comparisons between two groups were made using the Student's t-test. Statistical significance was set at $\mathrm{P}<0.05$.

\section{Results}

PNE induces depression-like symptoms in offspring rats. Behavioral data obtained from the open field test and sucrose preference test are presented in Fig. 1. The result showed that PNE significantly prolonged immobility time $(\mathrm{P}<0.05 ;$ Fig. $1 \mathrm{~A})$ and decreased the sucrose preference $(\mathrm{P}<0.05$; Fig. $1 \mathrm{C})$ in female rats compared to the control group. However, male rats did not show any significant changes in regards to immobility time and sucrose preference (Fig. 1A). The horizontal movement in the open field did not differ significantly between the control and PNE group ( $\mathrm{P}>0.05$; Fig. 1B) both in the male and female rats.

PNE induces hippocampal morphological changes in offspring rats. Hippocampus pathomorphology was evaluated using Nissl staining. As shown in Fig. 2A-C and G-I, in normal rat hippocampus, the pyramidal cells and the granular cells were arranged in a neat and dense manner. Compared 

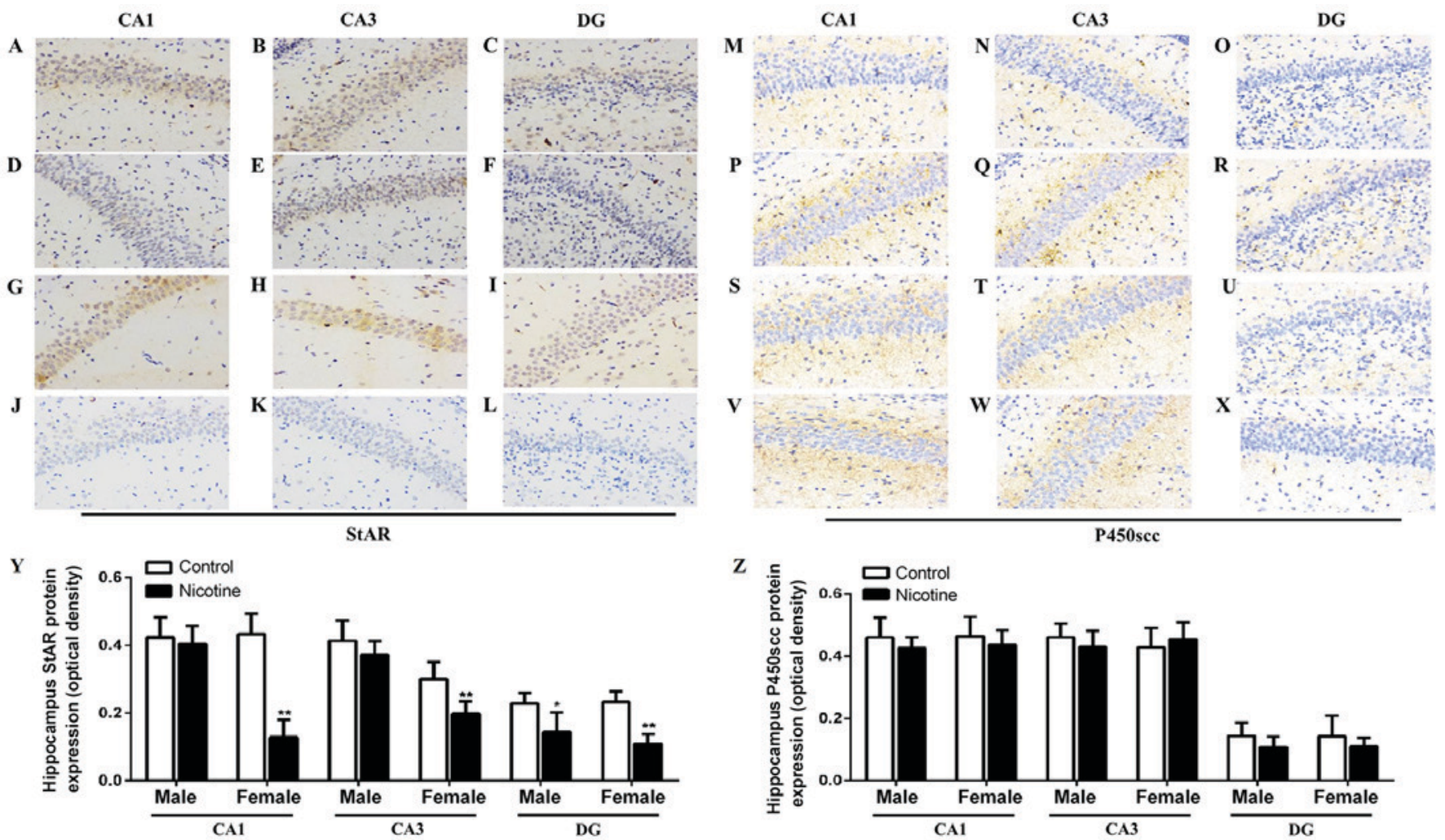

Figure 3. Effects of PNE on the expression of StAR and P450scc by immunohistochemistry and semi-quantitative analysis in hippocampus of adolescent rats. (A-C) Protein expression of StAR in male hippocampus in the control group (magnification, $x 40$ ). (D-F) Protein expression of StAR in male hippocampus in the PNE group (magnification, x40). (G-I) Protein expression of StAR in female hippocampus in the control group (magnification, x40). (J-L) Protein expression of StAR in female hippocampus in the PNE group (magnification, x40). (M-O) Protein expression of P450scc in male hippocampus in the control group (magnification, $\mathrm{x} 40$ ). (P-R) Protein expression of P450scc in male hippocampus in the PNE group (magnification, $\mathrm{x} 40$ ). (S-U) Protein expression of $\mathrm{P} 450 \mathrm{scc}$ in female hippocampus in the control group (magnification, $\mathrm{x} 40)$. (V-X) protein expression of P450scc in female hippocampus in the PNE group (magnification, $\mathrm{x} 40$ ). (Y and Z) quantification of StAR and P450scc from the immunohistochemical staining. Five sections of each group were selected and five random fields of each section scored. The data are expressed as mean \pm standard error of the mean; $n=4$ offsprings. ${ }^{*} \mathrm{P}<0.05,{ }^{* *} \mathrm{P}<0.01$ vs. control. PNE, prenatal nicotine exposure; StAR, steroidogenic acute regulatory protein; P450scc, P450 side-chain cleavage enzyme.

to that in the control group, the arrangement of cell layers in the PNE group was sparse and Nissl substance was decreased or dissolved (Fig. 2E and K). The numbers of hippocampal neurons were significantly decreased in PNE group rats, especially in the CA3 and DG regions of female rats $(\mathrm{P}<0.01$ and $\mathrm{P}<0.05$; Fig. $2 \mathrm{~N}$ and $\mathrm{O}$ ). Male rat offspring also showed decreased hippocampal neurons in the CA3 region compared with the control group animals, though the difference was not statistically significant $(\mathrm{P}=0.07$; Fig. $2 \mathrm{~N})$.

PNE decreases expression of hippocampal StAR/P450scc in offspring rats. StAR and $\mathrm{P} 450 \mathrm{scc}$ are essential for the production of steroid hormones including cortisol, aldosterone and dehydroepiandrosterone sulfate $(12,32)$. The protein expression of StAR/P450scc was determined using immunohistochemistry. Immunohistochemical analysis with anti-StAR (Fig. 3A-L) and anti-P450scc (Fig. 3M-X) specific antibody showed positive staining in the cytoplasm. Semi-quantitative assay results also indicated the decreased protein expression of StAR in the hippocampal CA1, CA3 and DG regions of PNE female rats compared to the control group, while StAR expression only decreased in the DG region of PNE male rats $(\mathrm{P}<0.05, \mathrm{P}<0.01$; Fig. $3 \mathrm{Y})$. No significant differences in the protein expression of $\mathrm{P} 450 \mathrm{scc}$ was observed in the hippocampus between the control and PNE rats (Fig. 3Z).
PNE induces hippocampal morphological changes in fetal rats. Fetal hippocampus pathomorphology in CA1, CA3 and DG regions were evaluated using Nissl staining. Compared to that in the control group, the cell layer in PNE rats exhibited irregular cell arrangement and light staining (Fig. 4D, E and L); the number of neurons was significantly decreased in the fetal hippocampus, especially in CA3 regions $(\mathrm{P}<0.05, \mathrm{P}<0.01$; Fig. 4N).

PNE promotes female fetal hippocampus steroidogenesis. ELISA and RT-qPCR were used to detect the content of CORT and the mRNA expression of StAR/P450scc in fetal hippocampus. Compared to control animals, female fetal rats from the PNE group showed increased CORT content and StAR expression in the hippocampus $(\mathrm{P}<0.05, \mathrm{P}<0.01)$, while no significant differences in CORT content and StAR expression were observed in the male fetal hippocampus (Fig. 5A, B and D). The mRNA and protein expression of P450scc did not differ in the PNE and control fetal hippocampus (Fig. 5C and D).

\section{Discussion}

Adverse events in brain development have lasting effects on brain function and emotional development (33-35), which 
A
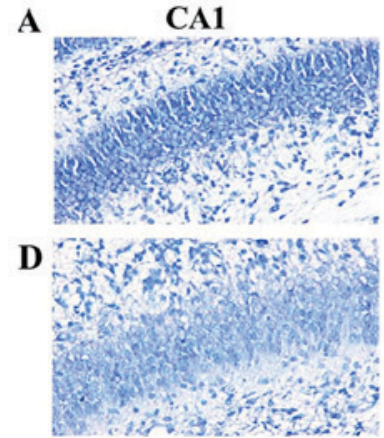

G

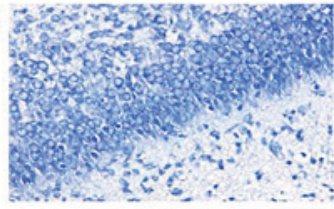

J

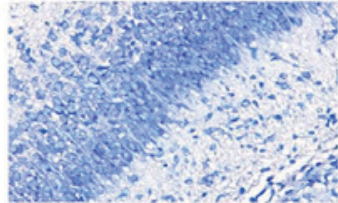

B

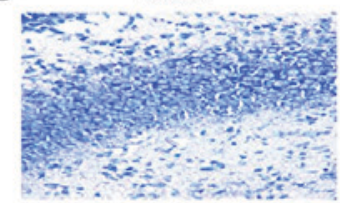

E

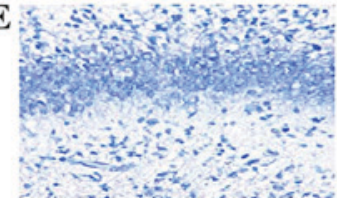

H

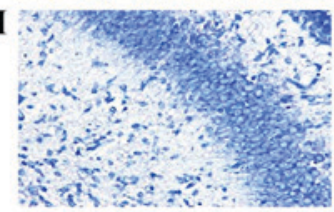

K

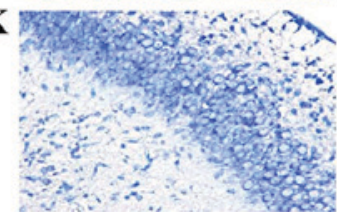

C

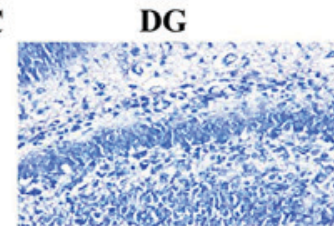

F

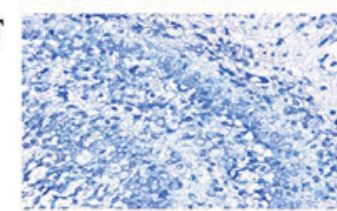

I

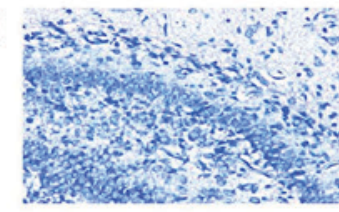

L

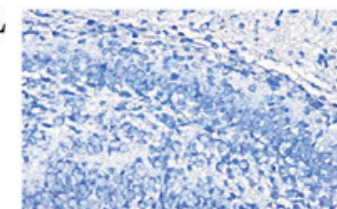

M

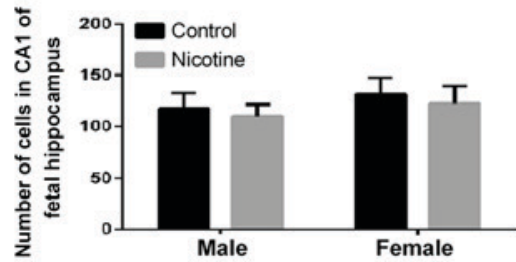

$\mathbf{N}$

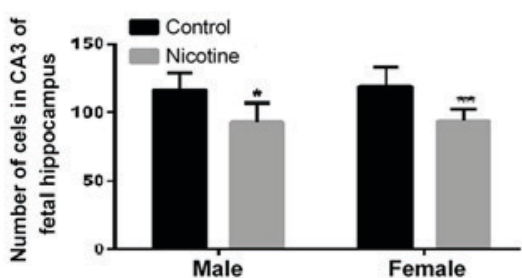

0

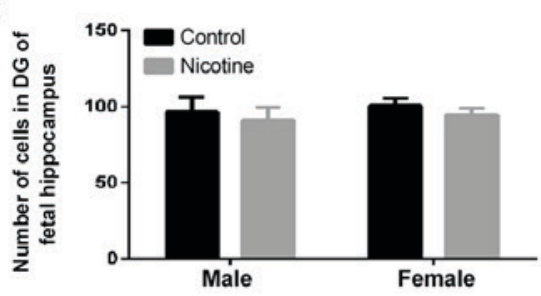

Figure 4. Morphological evaluation of Nissl staining in CA1, CA3 and DG regions of hippocampus in fetal rats. (A-C) Morphology of the hippocampus in the male control group (magnification, x400). (D-F) Morphology of the hippocampus in the male PNE group (magnification, x400). (G-I) Morphology of the hippocampus in the female control group (magnification, x400). (J-L) Morphology of the hippocampus in the female PNE group (magnification, x400). (M-O) Number of cells in CA1, CA3 and DG of fetal rat hippocampus. Five sections of each group were selected and five random fields of each section scored. The data are expressed as mean \pm standard error of the mean, $\mathrm{n}=6$ pregnant rats; ${ }^{*} \mathrm{P}<0.05,{ }^{* *} \mathrm{P}<0.01$ vs. control. PNE, prenatal nicotine exposure.
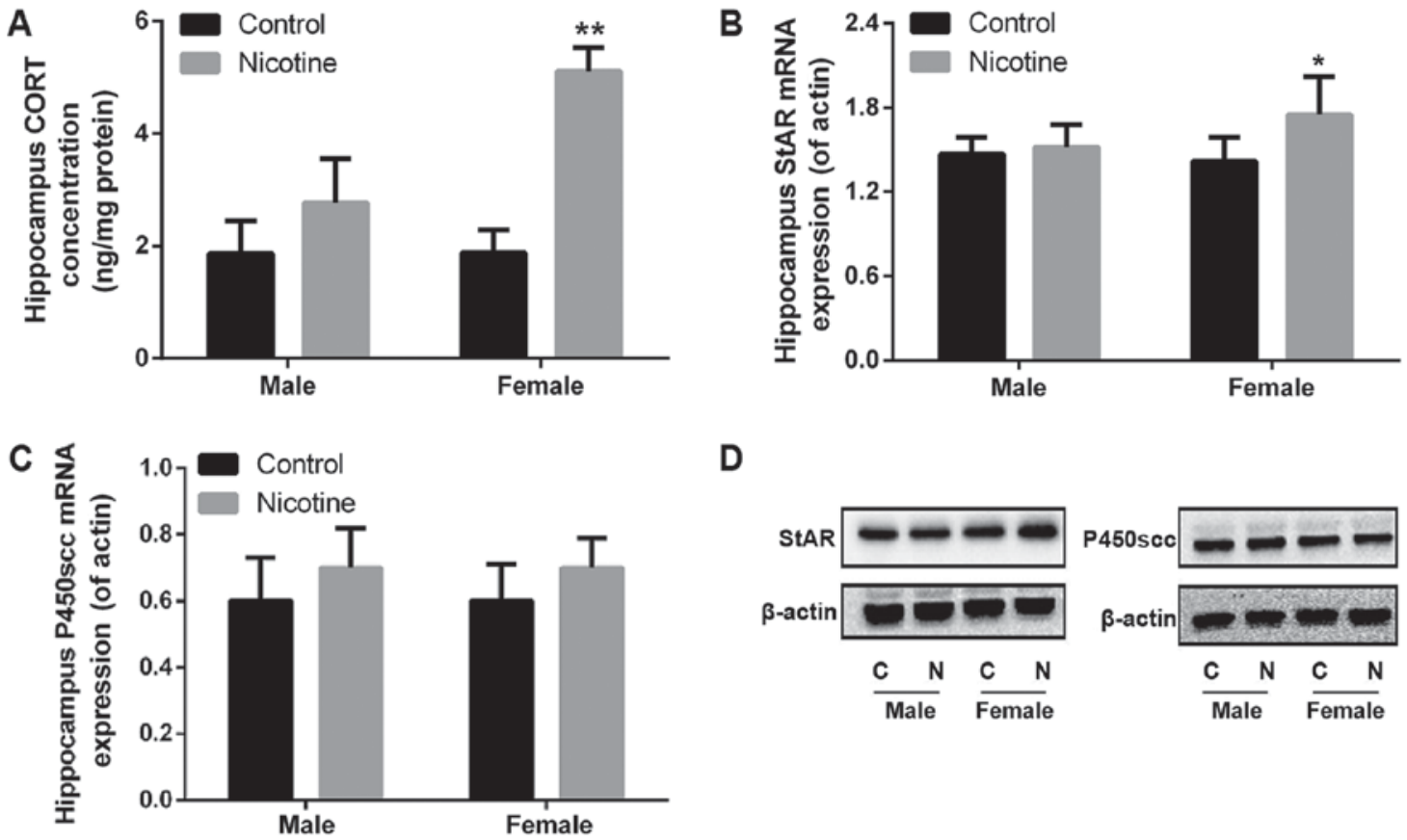

D

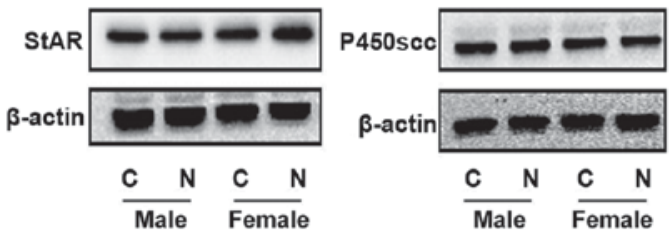

Figure 5. Effects of PNE on fetal hippocampus steroidogenesis. Pregnant rats were subcutaneously administered $1.0 \mathrm{mg} / \mathrm{kg}$ nicotine twice per day from GD9 to 20. The hippocampus was dissected and collected at GD20. The hippocampal samples from each litter were counted as one sample. (A) The level of hippocampus CORT content was determined using ELISA. (B) The mRNA expression of StAR was assessed using RT-qPCR. (C) The mRNA expression of P450scc was assessed using RT-qPCR. (D) StAR and P450scc protein expression was assessed via western blot analysis. The data are expressed as mean \pm standard error of the mean; $n=6$ pregnant rats. ${ }^{*} \mathrm{P}<0.05,{ }^{* *} \mathrm{P}<0.01$ vs. control. PNE, prenatal nicotine exposure; GD, gestational day; CORT, corticosterone; StAR, steroidogenic acute regulatory protein; $\mathrm{P} 450 \mathrm{scc}, \mathrm{P} 450$ side-chain cleavage enzyme.

may result in the increase of the sensitivity to the progression of mood disorders $(8,36)$. Gestational exposure to many xenobiotics, such as drugs and environmental chemicals, is able to affect the immature neural circuitry and increase the susceptibility to depression $(5,27,37)$. Studies on both humans and animals confirmed that prenatal nicotine exposure (PNE) 
induced depressive symptoms in offspring $(8,38-40)$. In the present study, our data manifested the significant increase in immobility time in the open field test and the sucrose preference rate in the sucrose preference test in female offspring rats exposed to nicotine during pregnancy. This finding suggested that PNE could induce the depression-like symptom in adolescent female rats. The limitation of this study is that the open space test is often used to assess depressive behavioral changes, yet, the tail suspension test may be better to observe the immobility time. Although our previous studies confirmed that PNE induced intrauterine growth retardation (IUGR) $(21,41)$, which were referred to as developing baby weighs $10 \%$ or two standard deviations less than the mean body weight of normal babies at the same gestational age (42). As the model of nicotine exposure during pregnancy is relatively stable $(21,22)$, the weight of offspring was not included in this experiment.

The hippocampus exerts an important function in cognition and mood regulation by modulating anxiety states and depression (43). The gestational period of rats spans 21 days, which is developmentally equivalent to the first two trimesters of human gestation. Many critical developmental events occur during this period, including the appearance of nicotinic acetylcholine receptors (nAChRs) (44), the generation of most neuronal cell groups and early synaptogenesis (45). Postnatal development of rats and mice in the first 4 weeks is a crucial phase during which DG neurogenesis and granule cell maturation occur $(46,47)$. In order to focus on the critical time, the period of PNE was selected from GD9 to GD20 and brain samples were collected at PD35 in our animal model. Research has found that PNE suppresses DNA synthesis in rat brains (48), reduces fetal brain weight when pregnant rats are treated with nicotine from early and middle gestation (34). PNE increased the expression of mRNA of fetal brain $\alpha 7$ $\mathrm{nAChRs}$ (34); $\alpha 7 \mathrm{nAChRs}$ are associated with the increased cell death of immature neurons (49). Thus, $\alpha 7 \mathrm{nAChRs}$ could increase the vulnerability to the cytotoxic effects from nicotine in immature neurons. In the present study, the Nissl staining results showed that the fetal hippocampal CA3 region exhibited pathomorphological changes in PNE offspring, which lasted to the adolescent period. Our observation was in agreement with previous studies which demonstrated that PNE induces pathomorphological changes in infants and offspring (50,51). Loss of cells in the central nervous system could be due to various reasons, including reduced proliferation, loss of progenitor pools, or increased apoptosis due to neurotoxic agents $(52,53)$. In the present study, we observed loss of hippocampal cells in the CA3 and DG regions of female progenies; however, the female fetus exhibited a decreased number of cells in CA3, but not DG. This evidence suggested that progenitor cell numbers are not affected in general, but the loss of mature neurons of CA3 could be the reason for the behavioral changes observed. Secondary neurogenesis of the rat hippocampus has not yet reached maturity by P29 or P35 (47); in the present study, the existence of delayed brain development could not be confirmed in the PNE group, as various marker proteins, which are expressed during distinct periods of neurodevelopment, were not detected.

Neurosteroids play a key role in depression. Animal studies have demonstrated that fluoxetine, which is widely used as an antidepressant, could enhance the levels of allopregnanolone (neuroactive steroid) in rat brain (54), while direct administration of allopregnanolone alleviated depressive behaviors in animal models of depression (55). Recent studies have found that overexpression of hippocampal translocator protein (TSPO), which functions in the shuttling of cholesterol to the inner mitochondrial membrane, produced antidepressant-like behavioural effects in mice (56). The result suggested that TSPO-mediated allopregnanolone synthesis is associated with depression. Previous research has shown that CORT synthesized in hippocampal neurons may play a role in the modulation of synaptic plasticity (57). Few studies have looked into the relation of PNE and CORT in the hippocampus. The brain expresses the rate-limiting enzyme of steroidogenesis, StAR and P450scc, which are essential for the production of steroid hormones including cortisol, aldosterone and dehydroepiandrosterone sulfate (DHEAS) $(58,59)$. StAR mediates the translocation of cholesterol from the outer to the inner mitochondrial membrane, which is the initial and rate-limiting step in adrenocortical steroid biosynthesis, while P450scc cleaves the cholesterol side chain, converting cholesterol to pregnenolone, the precursor of androgens, estrogens and progesterone (59). Our data showed that PNE suppressed StAR expression in CA1, CA3 and DG regions of adolescent female rats and only in DG regions of adolescent male rats. However, there was differential change that the level of CORT and the expression of StAR in female fetal hippocampus of the PNE group were increased. The result implies that PNE should decrease the cholesterol transport from the outer to the inner mitochondrial membrane and StAR expression changes in DG regions of adolescent male rats are not related to behavioral changes. Although the expression of StAR proteins in the fetal or adolescent hippocampus was detected respectively by western blotting and immunohistochemistry, it was not the reason for the inconsistent expression changes of StAR in fetal and adolescent stages. We prefer to do immunohistochemistry to observe changes in protein expression, because it can clearly assess the protein change in different region. Due to limited samples, we regret that we did not do immunohistochemistry of the fetal hippocampus. PNE transiently increased StAR and CORT in the fetal hippocampus, which may be a feedback neuroprotective mechanism against neurotoxic effects of nicotine. After birth, increased $\alpha 7 \mathrm{nAChRs}$ consistently caused neuronal injury during the period of hippocampal neurogenesis. At this point we can only speculate that PNE-induced change in StAR and CORT in the rat hippocampus is associated with offspring depression-like behavior.

It is known that depression is affected by sex, age and hormonal status in humans and animals. Women have a higher prevalence of depression than men in general, but the differences fade away slowly in aged populations $(60,61)$. Brain-derived neurotrophic factor (BDNF)-knockout mice were used as another model of depression and showed marked gender differences. Female rats but not male rats exhibited depression-related behaviors (62). The study on sex-dependent effects of nicotine on developmental brain mainly focus on the nAChR and sex hormones (63). Some studies suggest that sex-based differences in depression may result from alterations in hormonal levels (64). Previous research has demonstrated that females are more sensitive than males to the effects of nicotine (65). Evidence suggests that nicotine/nAChR has 
crosstalk with estrogen or estrogen receptors $(66,67)$. In this study, we only found that PNE induced depression-like behavior in female adolescent rats but not in male rats, and that the level of CORT in the female hippocampus was different from that noted in the male. PNE was found to suppress StAR expression in the $\mathrm{CA} 3$ region of adolescent female rats and not male rats. Therefore, we speculate that the behavioral and histopathological changes in only female mice due to nicotine were via StAR expression in the CA3 region. Taken together, we found a sex difference in PNE offspring rats and demonstrated that female offspring rats were more susceptible to depression during PNE. However, the complicated molecular mechanisms are far from clear, and further investigation is needed.

In conclusion, the common gestational exposure factor, nicotine, was used as our study model, and the findings demonstrated that PNE caused damage to hippocampus neurons, led to neurosteroid disorders in the hippocampus of fetal and adolescent female rats, and enhanced depression-like behaviors in female adolescent rats. We speculate that the neurosteroid level is the primary reason for the depression-like behavior changes observed in female progenies. These sex differences via neurosteroid level may confer sex-biased risk for those related to mental health. Our results provide further insight into our understanding of nicotine developmental toxicity.

\section{Acknowledgements}

The authors would like to thank Dr Wang Hong (Department of Pharmacology, National University of Singapore) for assistance in editing the manuscript.

\section{Funding}

The present study was supported by grants from the Nature Science Foundation of Hubei Province (grant no. 2018CFB667), and the Yangtze Fund for Youth Teams of Science and Technology Innovation (grant no. 2016cqt04).

\section{Availability of data and materials}

The datasets used and/or analyzed during the current study are available from the corresponding author on reasonable request.

\section{Authors' contributions}

$\mathrm{CZ}$ and SJF performed the majority of the experiments and drafted the manuscript. ABS performed the RT-qPCR assays and ZZL performed the corticosterone detection assays; ABS and ZZL contributed to the interpretation of data and revised the manuscript. LL designed the experiments and is responsibile for the integrity of the data and the accuracy of the data analysis.

\section{Ethics approval and consent to participate}

All animal studies were performed at the Animal Center of Yangtze University, accredited by the Association for Assessment and Accreditation of Laboratory Animal Care
International (AAALAC International; Frederick, MD, USA). All animal experiments were approved by the Ethics Committee of Yangtze University, School of Medicine (Jingzhou, China) and performed in accordance with the Guidelines for the Care and Use of Laboratory Animals of Yangtze University.

\section{Patient consent for publication}

Not applicable.

\section{Competing interests}

The authors declare that they have no competing interests.

\section{References}

1. Organization WH: Integrating mental health into primary care: A global perspective 2008 .

2. Liu Q, Cai H, Yang LH, Xiang YB, Yang G, Li H, Gao YT, Zheng W, Susser E and Shu XO: Depressive symptoms and their association with social determinants and chronic diseases in middle-aged and elderly Chinese people. Sci Rep 8: 3841, 2018.

3. Capra L, Tezza G, Mazzei F and Boner AL: The origins of health and disease: The influence of maternal diseases and lifestyle during gestation. Ital J Pediatr 39: 7, 2013.

4. Pierrefiche O: Long term depression in rat hippocampus and the effect of ethanol during fetal life. Brain Sci 7: E157, 2017.

5. Belluscio LM, Alberca CD, Pregi N and Canepa ET: Altered gene expression in hippocampus and depressive-like behavior in young adult female mice by early protein malnutrition. Genes Brain Behav 15: 741-749, 2016.

6. Tong VT, Dietz PM, Morrow B, D'Angelo DV, Farr SL, Rockhill KM and England LJ; Centers for Disease Control and Prevention (CDC): Trends in smoking before, during, and after pregnancy-Pregnancy Risk Assessment Monitoring System, United States, 40 sites, 2000-2010. MMWR Surveill Sum 62: 1-19, 2013.

7. Maughan B, Taylor A, Caspi A and Moffitt TE: Prenatal smoking and early childhood conduct problems: Testing genetic and environmental explanations of the association. Arch Gen Psychiatry 61: 836-843, 2004.

8. Fergusson DM, Woodward LJ and Horwood LJ: Maternal smoking during pregnancy and psychiatric adjustment in late adolescence. Arch Gen Psychiatry 55: 721-727, 1998.

9. Stene-Larsen K, Borge AI and Vollrath ME: Maternal smoking in pregnancy and externalizing behavior in 18-month-old children: Results from a population-based prospective study. J Am Acad Child Adolesc Psychiatry 48: 283-289, 2009.

10. Girdler SS and Klatzkin R: Neurosteroids in the context of stress: Implications for depressive disorders. Pharmacol Ther 116: 125-139, 2007.

11. Jukic MM, Opel N, Strom J, Carrillo-Roa T, Miksys S, Novalen M, Renblom A, Sim SC, Peñas-Lledó EM, Courtet P, et al: Elevated CYP2C19 expression is associated with depressive symptoms and hippocampal homeostasis impairment. Mol Psychiatry 22: 1224, 2017.

12. Reddy DS: Neurosteroids: Endogenous role in the human brain and therapeutic potentials. Prog Brain Res 186: 113-137, 2010.

13. Baulieu EE: Neurosteroids: A novel function of the brain. Psychoneuroendocrinology 23: 963-987, 1998.

14. Izumi Y, O'Dell KA and Zorumski CF: Corticosterone enhances the potency of ethanol against hippocampal long-term potentiation via local neurosteroid synthesis. Front Cell Neurosci 9: 254, 2015.

15. Tokuda K, Izumi Y and Zorumski CF: Ethanol enhances neurosteroidogenesis in hippocampal pyramidal neurons by paradoxical NMDA receptor activation. J Neurosci 31: 9905-9909, 2011.

16. Slotkin TA: If nicotine is a developmental neurotoxicant in animal studies, dare we recommend nicotine replacement therapy in pregnant women and adolescents? Neurotoxicol Teratol 30: 1-19, 2008.

17. Hellström-Lindahl E and Nordberg A: Smoking during pregnancy: A way to transfer the addiction to the next generation? Respiration 69: 289-293, 2002. 
18. Roy TS, Seidler FJ and Slotkin TA: Prenatal nicotine exposure evokes alterations of cell structure in hippocampus and somatosensory cortex. J Pharmacol Exp Ther 300: 124-133, 2002.

19. Li J, Bo L, Zhang P, Gao Q, Li L, Tang J, Wu C, Li D, Xiao J, Chen J, et al: Exposure to nicotine during pregnancy and altered learning and memory in the rat offspring. Nicotine Tob Res 17: 661-666, 2015.

20. Kalejaiye OO and Gondre-Lewis MC: Enhanced susceptibility of CA3 hippocampus to prenatal nicotine exposure. J Dev Orig Health Dis 8: 155-160, 2017.

21. Yan YE, Liu L, Wang JF, Liu F, Li XH, Qin HQ and Wang H: Prenatal nicotinic exposure suppresses fetal adrenal steroidogenesis via steroidogenic factor 1 (SF-1) deacetylation. Toxicol Appl Pharmacol 277: 231-241, 2014.

22. Liu L, Wang JF, Fan J, Rao YS, Liu F, Yan YE and Wang H: Nicotine suppressed fetal adrenal StAR expression via YY1 mediated-histone deacetylation modification mechanism. Int J Mol Sci 17: E1477, 2016.

23. Rice D and Barone S Jr: Critical periods of vulnerability for the developing nervous system: Evidence from humans and animal models. Environ Health Perspect 108 (Suppl 3): S511-S533, 2000

24. Semple BD, Blomgren K, Gimlin K, Ferriero DM and Noble-Haeusslein LJ: Brain development in rodents and humans: Identifying benchmarks of maturation and vulnerability to injury across species. Prog Neurobiol 106-107: 1-16, 2013.

25. Bayer SA: Development of the hippocampal region in the rat II. Morphogenesis during embryonic and early postnatal life. J Comp Neurol 190: 115-134, 1980

26. Schaefer TL, Skelton MR, Herring NR, Gudelsky GA, Vorhees CV and Williams MT: Short- and long-term effects of $(+)$-methamphetamine and (+/-)-3,4-methylenedioxymethamphetamine on monoamine and corticosterone levels in the neonatal rat following multiple days of treatment. J Neurochem 104: 1674-1685, 2008.

27. Zhang Y, Zhu X, Bai M, Zhang L, Xue L and Yi J: Maternal deprivation enhances behavioral vulnerability to stress associated with miR-504 expression in nucleus accumbens of rats. PLoS One 8: e69934, 2013.

28. Bowen MT, Peters ST, Absalom N, Chebib M, Neumann ID and McGregor IS: Oxytocin prevents ethanol actions at delta subunit-containing GABAA receptors and attenuates ethanol-induced motor impairment in rats. Proc Natl Acad Sci USA 112: 3104-3109, 2015.

29. Gao J, Wang H, Liu Y, Li YY, Chen C, Liu LM, Wu YM, Li S and Yang C: Glutamate and GABA imbalance promotes neuronal apoptosis in hippocampus after stress. Med Sci Monit 20: 499-512, 2014

30. Pan M and Zhang C: Stimulatory effect of gonadal hormones on fetal rat hippocampal neural proliferation requires neurotrophin receptor activation in vitro. Neurosci Lett 546: 1-5, 2013.

31. Livak KJ and Schmittgen TD: Analysis of relative gene expression data using real-time quantitative PCR and the 2(-Delta Delta C(T)) method. Methods 25: 402-408, 2001.

32. Sierra A: Neurosteroids: The StAR protein in the brain. J Neuroendocrinol 16: 787-793, 2004.

33. Teicher MH and Samson JA: Annual research review: Enduring neurobiological effects of childhood abuse and neglect. J Child Psychol Psychiatry 57: 241-266, 2016.

34. Lv J, Mao C, Zhu L, Hong Zhang, Hui Pengpeng, Feichao Xu, Yujuan Liu, Lubo Zhang and Zhice Xu: The effect of prenatal nicotine on expression of nicotine receptor subunits in the fetal brain. Neurotoxicology 29: 722-726, 2008.

35. Krugers HJ, Arp JM, Xiong H, Kanatsou S, Lesuis SL, Korosi A, Joels M and Lucassen PJ: Early life adversity: Lasting consequences for emotional learning. Neurobiol Stress 6: 14-21, 2017.

36. Chudal R, Brown AS, Gissler M, Suominen A and Sourander A Is maternal smoking during pregnancy associated with bipolar disorder in offspring? J Affect Disord 171: 132-136, 2015.

37. Martinez EJ,Kolb BL, Bell A, Savage DD and Allan AM: Moderate perinatal arsenic exposure alters neuroendocrine markers associated with depression and increases depressive-like behaviors in adult mouse offspring. Neurotoxicology 29: 647-655, 2008.

38. Sobrian SK, Marr L and Ressman K: Prenatal cocaine and/or nicotine exposure produces depression and anxiety in aging rats. Prog Neuropsychopharmacol Biol Psychiatry 27: 501-518, 2003.

39. Menezes AM, Murray J, Laszlo M, Wehrmeister FC, Hallal PC, Gonçalves H, Assunção MC, Menezes CB and Barros FC: Happiness and depression in adolescence after maternal smoking during pregnancy: Birth cohort study. PLoS One 8: e80370, 2013.
40. Elmasry H, Goodwin RD, Terry MB and Tehranifar P: Early life exposure to cigarette smoke and depressive symptoms among women in midlife. Nicotine Tob Res 16: 1298-1306, 2014.

41. Xu D, Liang G, Yan YE, He WW, Liu YS, Chen LB, Magdalou J and Wang $\mathrm{H}$ : Nicotine-induced over-exposure to maternal glucocorticoid and activated glucocorticoid metabolism causes hypothalamic-pituitary-adrenal axis-associated neuroendocrine metabolic alterations in fetal rats. Toxicol Lett 209: 282-290, 2012.

42. Silveira VM and Horta BL: Birth weight and metabolic syndrome in adults: Meta-analysis. Rev Saude Publica 42: 10-18, 2008 (In Portuguese).

43. McEwen BS, Bowles NP, Gray JD, Hill MN, Hunter RG, Karatsoreos IN and Nasca C: Mechanisms of stress in the brain. Nat Neurosci 18: 1353-1363, 2015.

44. Naeff B, Schlumpf M and Lichtensteiger W: Pre- and postnata development of high-affinity $[3 \mathrm{H}]$ nicotine binding sites in rat brain regions: An autoradiographic study. Brain Res Dey Brain Res 68: 163-174, 1992.

45. Bayer SA, Altman J, Russo RJ and Zhang X: Timetables of neurogenesis in the human brain based on experimentally determined patterns in the rat. Neurotoxicology 14: 83-144, 1993.

46. Quinn R: Comparing rat's to human's age: How old is my rat in people years? Nutrition 21: 775-777, 2005.

47. Radic T, Friess L, Vijikumar A, Jungenitz T, Deller T and Schwarzacher SW: Differential postnatal expression of neuronal maturation markers in the dentate gyrus of mice and rats. Front Neuroanat 11: 104, 2017.

48. Slotkin TA, Greer N, Faust J, Cho H and Seidler FJ: Effects of maternal nicotine injections on brain development in the rat: Ornithine decarboxylase activity, nucleic acids and proteins in discrete brain regions. Brain Res Bull 17: 41-50, 1986.

49. Laudenbach V,Medja F,Zoli M, Rossi FM, Evrard P, Changeux JP and Gressens P: Selective activation of central subtypes of the nicotinic acetylcholine receptor has opposite effects on neonatal excitotoxic brain injuries. FASEB J 16: 423-425, 2002.

50. Roy TS and Sabherwal U: Effects of gestational nicotine exposure on hippocampal morphology. Neurotoxicol Teratol 20: 465-473, 1998

51. Wang $\mathrm{H}$ and Gondre-Lewis MC: Prenatal nicotine and maternal deprivation stress de-regulate the development of CA1, CA3, and dentate gyrus neurons in hippocampus of infant rats. PLoS One 8: e65517, 2013

52. Lugert S, Basak O, Knuckles P, Haussler U, Fabel K, Götz M, Haas CA, Kempermann G, Taylor V and Giachino C: Quiescent and active hippocampal neural stem cells with distinct morphologies respond selectively to physiological and pathological stimuli and aging. Cell Stem Cell 6: 445-456, 2010.

53. Shruster A, Melamed E and Offen D: Neurogenesis in the aged and neurodegenerative brain. Apoptosis 15: 1415-1421, 2010.

54. Fry JP, Li KY, Devall AJ, Cockcroft S, Honour JW and Lovick TA: Fluoxetine elevates allopregnanolone in female rat brain but inhibits a steroid microsomal dehydrogenase rather than activating an aldo-keto reductase. Br J Pharmacol 171: 5870-5880, 2014.

55. Khisti RT and Chopde CT: Serotonergic agents modulate antidepressant-like effect of the neurosteroid 3alpha-hydroxy-5al pha-pregnan-20-one in mice. Brain Res 865: 291-300, 2000.

56. Li L, Wang W, Zhang LM, Jiang XY, Sun SZ, Sun LJ, Guo Y, Gong J, Zhang YZ, Wang HL and Li YF: Overexpression of the $18 \mathrm{kDa}$ translocator protein (TSPO) in the hippocampal dentate gyrus produced anxiolytic and antidepressant-like behavioural effects. Neuropharmacology 125: 117-128, 2017.

57. Higo S, Hojo Y, Ishii H, Komatsuzaki Y, Ooishi Y, Murakami G, Mukai H, Yamazaki T, Nakahara D, Barron A, et al: Endogenous synthesis of corticosteroids in the hippocampus. PLoS One 6: e21631, 2011.

58. Tsutsui K, Ukena K, Usui M, Sakamoto H and Takase M: Novel brain function: Biosynthesis and actions of neurosteroids in neurons. Neurosci Res 36: 261-273, 2000.

59. Manna PR and Stocco DM: Regulation of the steroidogenic acute regulatory protein expression: Functional and physiological consequences. Curr Drug Targets Immune Endocr Metabol Disord 5: 93-108, 2005 .

60. Solomon MB and Herman JP: Sex differences in psychopathology: Of gonads, adrenals and mental illness. Physiol Behav 97: 250-258, 2009.

61. Xu Y, Ma L, Jiang W, Li Y, Wang G and Li R: Study of sex differences in duloxetine efficacy for depression in transgenic mouse models. Front Cell Neurosci 11: 344, 2017. 
62. Autry AE, Adachi M, Cheng $\mathrm{P}$ and Monteggia LM: Gender-specific impact of brain-derived neurotrophic factor signaling on stress-induced depression-like behavior. Biol Psychiatry 66: 84-90, 2009.

63. Cross SJ, Linker KE and Leslie FM: Sex-dependent effects of nicotine on the developing brain. J Neurosci Res 95: 422-436, 2017.

64. Worly BL, Gur TL and Schaffir J: The relationship between progestin hormonal contraception and depression: A systematic review. Contraception 97: 478-489, 2018.

65. O'Dell LE and Torres OV: A mechanistic hypothesis of the factors that enhance vulnerability to nicotine use in females. Neuropharmacology 76: 566-580, 2014.
66. Lee $\mathrm{CH}$, Chang YC, Chen CS, Tu SH, Wang YJ, Chen LC, Chang YJ, Wei PL, Chang HW, Chang $\mathrm{CH}$, et al: Crosstalk between nicotine and estrogen-induced estrogen receptor activation induces alpha9-nicotinic acetylcholine receptor expression in human breast cancer cells. Breast Cancer Res Treat 129: 331-345, 2011.

67. el-Mas MM, el-Gowilly SM, Gohar EY, Ghazal AR and Abdel-Rahman AA: Estrogen dependence of the renal vasodilatory effect of nicotine in rats: Role of alpha7 nicotinic cholinergic receptor/eNOS signaling. Life Sci 88: 187-193, 2011.

(i)(9) This work is licensed under a Creative Common Attribution-NonCommercial-NoDerivatives 4.0 International (CC BY-NC-ND 4.0) License. 